\title{
Alejandro Magno, el "nuevo" Posidipo y los prodigios. A propósito de los epigramas 31 y 35 A.- B.
}

Alexandre le Grand, le «nouveau » Posidippe et les prodiges. À propos des épigrammes 31 et 35 A.-B.

Alexander the Great, the "New" Posidippus and the Miracles. About Epigrams 31 and 35 A.-B.

\section{Esteban Calderón Dorda}

\section{(2) OpenEdition}

Journals

Edición electrónica

URL: https://journals.openedition.org/gaia/2025

DOI: 10.4000/gaia.2025

ISSN: 2275-4776

Editor

UGA Éditions/Université Grenoble Alpes

Edición impresa

ISBN: 978-2-37747-292-5

ISSN: 1287-3349

Referencia electrónica

Esteban Calderón Dorda, "Alejandro Magno, el "nuevo" Posidipo y los prodigios. A propósito de los epigramas 31 y 35 A.-B. », Gaia [En ligne], 24 | 2021, mis en ligne le 31 mai 2021, consulté le 09 décembre 2021. URL : http://journals.openedition.org/gaia/2025 ; DOI : https://doi.org/10.4000/gaia 2025

Este documento fue generado automáticamente el 9 diciembre 2021.

Gaia. Revue interdisciplinaire sur la Grèce archaïque 


\section{Alejandro Magno, el "nuevo" Posidipo y los prodigios. A propósito de los epigramas 31 y 35 A.-B.}

Alexandre le Grand, le «nouveau » Posidippe et les prodiges. À propos des

épigrammes 31 et 35 A.-B.

Alexander the Great, the "New" Posidippus and the Miracles. About Epigrams 31 and 35 A.-B.

Esteban Calderón Dorda

\section{NOTA DEL AUTOR}

Este trabajo ha sido realizado en el marco del Proyecto de Investigación FFI2017-84036P del MICINN del Gobierno de España, titulado «Estudios sobre el "nuevo" Posidipo: elaboración de una nueva edición crítica y primera traducción en lengua española (con comentario)».

1 La portentosa vida de Alejandro Magno, que a menudo gravita entre la historia y el mito, siempre ofrece aspectos novedosos sobre los que centrar la reflexión filológica e histórica. Este es el caso de dos epigramas pertenecientes al llamado "nuevo" Posidipo, hallado en el P. Mil. Vogl. VIII 309, de Milán, en concreto los epigramas 31 y 35 A.-B., pertenecientes a la sección del papiro titulada oi $\omega v 0 \sigma \kappa o \pi ı \alpha ́$, esto es, 'augurios' ${ }^{1}$, de los que ofrezco aquí un nuevo texto y traducción, que discrepa en algunos aspectos de la edición de Austin-Bastianini². La piedad religiosa, así como la $\delta \varepsilon 1 \sigma ı \delta \alpha \imath \mu o v i ́ \alpha$, son una constante en la vida de Alejandro ${ }^{3}$ y sus decisiones, que están marcadas indeleblemente por los sucesos de tipo sobrenatural ${ }^{4}$. Esto es relevante, porque, al igual que en los

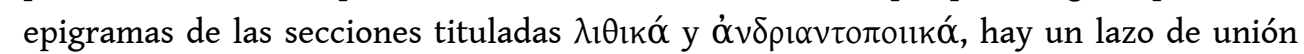
entre el contexto cultural de la corte ptolemaica del s. III a.C. y aquello que parece

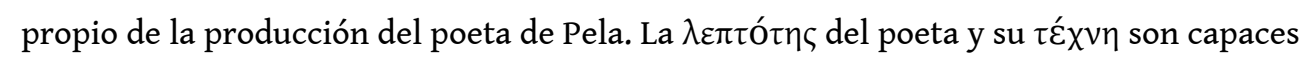




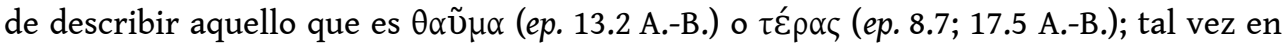
este sentido habría que entender el $\beta \tilde{\omega} \lambda$ os del epigrama 16 A.-B., que solamente la

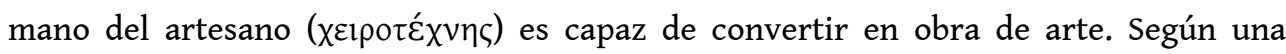
lectura geográfica bastante convincente ${ }^{5}$, los epigramas $\lambda_{l} \theta_{1} k \alpha$ a hacen un recorrido por todas las partes del mundo conquistado por Alejandro Magno: desde la India hasta la propia Grecia, entendiendo que son mencionadas sólo las regiones sometidas al imperio lágida, pero también aquellas donde los Lágidas extendían su influencia de manera más o menos cercana. Por otra parte, es posible observar la ausencia de alusiones claras a la herencia del Egipto antiguo, donde era conocida toda suerte de prácticas y de leyendas acerca de piedras mágicas, algo que se puede entender como un indicio de la voluntad de hacer del rey lágida el heredero de un imperio universal y no sólo el heredero de los faraones egipcios. Otro tanto sucede con el famoso epigrama de Lisipo (ep. 65 A.-B.) dedicado a Alejandro ${ }^{6}$ y que ya era conocido por la Antología Griega (XVI, 119), donde este epigrama inicia una serie de cuatro dedicados a Alejandro Magno. El epigrama revela cómo Lisipo había modelado una escultura con el semblante de Alejandro. La mirada del soberano se habría plasmado en el bronce con tal rasgo de verosimilitud $(\dot{\alpha} \lambda \eta \dot{\theta} \theta \varepsilon ı \alpha)$-según el 'canon de la verdad'- que se podía advertir en ella la llama de su ardor leonino. El interés de Posidipo por Lisipo podría estar motivado por el deseo de los monarcas helenísticos - los Ptolomeos en este caso- de mantener un nexo visual y artístico de unión con Alejandro ${ }^{7}$. En definitiva, en la Alejandría de los Ptolomeos las obras de arte, ya sean esculturas o gemas, son vistas en su relación con los Lágidas, en una sociedad en la que la corte real es el epicentro de toda actividad.

Veamos los epigramas que aquí nos ocupan.

El epigrama 31 ofrece el siguiente texto:

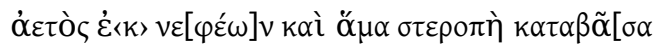

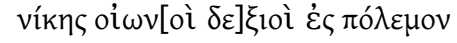

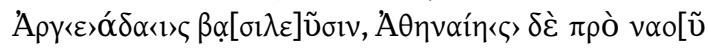

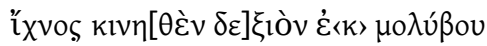

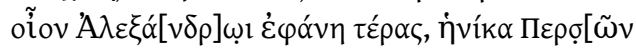

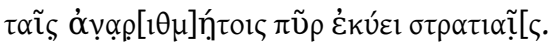

Un águila y un relámpago descendiendo de las nubes a la vez son augurios favorables de victoria para la guerra

para los reyes Argéadas, mas el pie derecho de Atenea [movido]

de la base de plomo delante del templo

se manifestó como un prodigio sólo a Alejandro, cuando preparaba 5

fuego contra los incontables ejércitos de los persas.

El epigrama comienza, como en otros casos, presentando el ave propicia para el augurio, pero en esta ocasión introduce una novedad al asociar el águila con el relámpago como signo de victoria. El águila es un ave destacada en la adivinación, además del ave de la realeza, y el rayo es símbolo de poder: en un contexto bélico ambos representaban buenos augurios, ya que se asociaban a Zeus ${ }^{8} \mathrm{y}$ no hay que olvidar la supuesta filiación divina de Alejandro. En Homero, Ilíada, XII, 353 y IX, 236, el relámpago es signo de victoria'; también el águila aparece como el mejor de los augurios en Ilíada, VIII, 247 y XII, $201^{10}$. Curcio Rufo ${ }^{11}$ se refiere a un águila que en la batalla de Gaugamela (331 a.C.) se apareció a Alejandro anunciando su triunfo, signo que renovó los ánimos de las tropas ${ }^{12}$. También Teócrito ${ }^{13}$ narra que un águila chilló tres veces desde lo alto, como augurio propicio, en el nacimiento de Ptolomeo II 
Filadelfo. La manifestación de ambos augurios refuerza el valor de los dos, como recuerda Cicerón, Sobre la adivinación, I, 106: partibus intonuit caeli pater ipse sinistris. / sic aquilae clarum firmavit Iuppiter omen. De ahí que Quinto de Esmirna ${ }^{14}$ asocie truenos y relámpagos a águilas que chillan denodadamente, como signos oủк $\dot{\alpha} \tau \varepsilon ́ \lambda \varepsilon \sigma \tau \alpha$ de la futura victoria de los griegos en Troya. Además, el rayo y el águila también están representados juntos en las monedas de los Ptolomeos, por lo que se ha pensado que la referencia a la dinastía Argéada puede ser un intento por vincular a esta familia con la de los Ptolomeos, que serían sus sucesores en Egipto y como uno de los aspectos de la propaganda vinculada con la figura de Alejandro y los presagios favorables para los monarcas Argéadas ${ }^{15}$. A la versión que ofrecerá Ps.-Calístenes (III, 33), Posidipo agrega el elemento del rayo, que, al igual que el águila, en la cultura helenística era un símbolo ideológico, ya que un águila enviada por Zeus había alimentado con su sangre al niño Ptolomeo, abandonado por su padre Lagos, cuando este supo que, en realidad, era hijo de Filipo I $^{16}$, de manera que simboliza la protección de la casa de los Ptolomeos ${ }^{17}$.

Hallamos una secuencia análoga a ó $\varepsilon \tau o ̀ \varsigma ~ \dot{\varepsilon}\langle\kappa\rangle v \varepsilon[\varphi \varepsilon ́ \omega] v$ en el comienzo de un pentámetro en un epigrama de Antípatro de Tesalónica ${ }^{18}$. De manera similar Teócrito

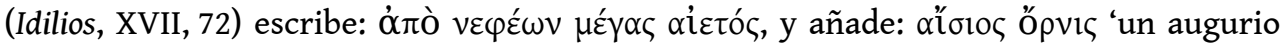
favorable', si bien el poeta juega aquí con la ambigüedad calculada del término ǒ $\rho v ı$ como 'augurio' y como 'ave'. A propósito del participio $\kappa \alpha \tau \alpha \beta \tilde{\alpha}[\sigma \alpha$ (v. 1), participium

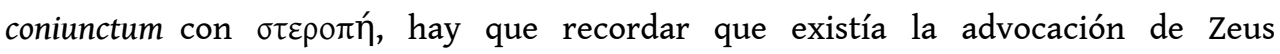

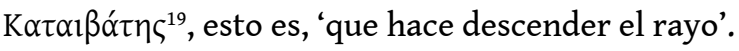

6 La dinastía de los Argéadas (v. 3), cuyo último soberano fue Alejandro Magno, gobernó Macedonia desde el s. vi al IV a.C., si bien la familia -no la dinastía- continuaría con Filipo Arrideo, hijo de Filipo II y Eurídice, Alejandro, hijo de Alejandro y Roxana, Heracles, hijo ilegítimo de Alejandro, y otros hijos de Filipo II. Según el epigrama de Posidipo, el macedonio recibió un signo excepcional antes de una excepcional victoria, la de Gaugamela, ante los persas ${ }^{20}$. Las hazañas de Alejandro, que habitualmente aparece en los epigramas del papiro en estrecha relación con los Ptolomeos, serán de nuevo recordadas en el ep. 35 A.-B. Hay que señalar que el papiro transmite $\alpha \rho \gamma \alpha \delta \alpha \varsigma$, corregido por Bastianini-Gallazzi ${ }^{21}$ en A $\rho \gamma\langle\varepsilon>\alpha ́ \alpha \alpha<\iota \varsigma$, , ya que no es raro que el copista presente dificultades con los nombres propios: ep. 14.3; 19.9; 34.2; 46.2; 51.3; 55.2; 62.4.5; 64.2; 84.2 A.-B.

7 Como se puede observar (supra), acepto en el texto (v.3) la lectura de Lapini ${ }^{22}$,

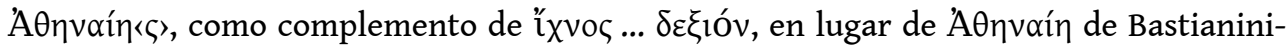
Gallazzi y preferible al dativo agente Änvaín〈l〉 - la iota adscrita es omitida con frecuencia en el papiro- de Ferrari ${ }^{23}$. Acepto también la primera versión de la partícula $\delta \varepsilon ́$ como adversativa, que ofrecen Bastianini-Gallazzi (2001), frente a la conectiva que introducen Austin-Bastianini (2002), de suerte que interpreto así el texto: «mas el pie derecho de Atenea [movido] de la base de plomo delante del templo [...]».

Posidipo introduce en el v. 4 una variatio en la estructura de este tipo de epigramas al

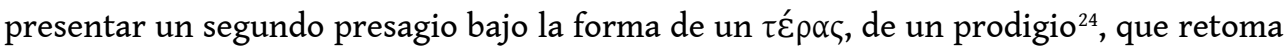
el tema de las estatuas, como en el ep. 30 A.-B., donde aparece una estatua de exuda.

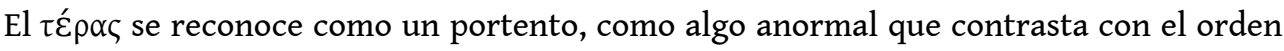
natural, a lo que hay que añadir que el movimiento de la estatua de una divinidad es, en general, un signo infausto, que anuncia una derrota ${ }^{25}$. Por el contrario, en algunos autores es un anuncio de victoria ${ }^{26}$, como en el caso de Suetonio (Vespasiano, V, 6): 
statuam Divi Iuli ad Orientem sponte conversam. En el caso que nos ocupa se trataba de un buen augurio para Alejandro -el adjetivo $\delta \varepsilon \xi ı$ ı́v conserva su valor de 'derecho', el lado más propicio para los presagios ${ }^{27}-$, si bien no se precisa el contexto en el que sucedió este hecho, tal vez porque era sobradamente conocido entre los hipotéticos lectores, aunque el prodigio no esté documentado. Por otra parte, parece lógico que un acontecimiento tan importante como la victoria de Alejandro sobre el poderoso ejército persa fuera preanunciado por un signo igualmente extraordinario. Se ha significado el hecho de que la pesadez del pie de Atenea indicaría la dificultad de la empresa ${ }^{28}$, pero, al mismo tiempo, el epigramista pone de relieve que la diosa, al mover el pie derecho, se pondrá en marcha al lado de Alejandro contra los persas. Lapini ${ }^{29}$ y Schröder ${ }^{30}$ sostienen que tuvo lugar antes de la campaña de Alejandro contra los persas, mientras que Tammaro ${ }^{31}$ propone como ocasión del epigrama la tercera guerra siríaca (246 a.C.), también aludida por Calímaco en su Cabellera de Berenice (fr. 110 Pfeiffer). Por su parte, Bastianini-Gallazzi ${ }^{32}$ defienden la posibilidad de que se tratase de la estatua de Atenea $\Pi \rho o v \alpha i ́ \alpha$, en el santuario apolíneo de Delfos ${ }^{33}$, posiblemente con motivo de la consulta que el macedonio hizo al oráculo antes de partir contra los persas, hecho que conocemos por Plutarco ${ }^{34}$, y en cuya línea van las conjeturas חpovó́o[v], de Lapini ${ }^{35} \mathrm{y}$ Прóv $\alpha$ [ $\varsigma]$, de Tammaro ${ }^{36}$, o tal vez una imagen que, de manera excepcional, estuviera ubicada en el exterior de un templo. Gronewald ${ }^{37}$ juega con la hipótesis de un completo desplazamiento de la estatua de Atenea desde el interior del templo y transferida $\pi \rho o ̀$ $v \alpha o v$. En este sentido, Plutarco ${ }^{38}$ recuerda que una de las primeras cosas que hizo Alejandro al cruzar el Helesponto fue ofrecer un sacrificio en honor de Atenea, en Ilión.

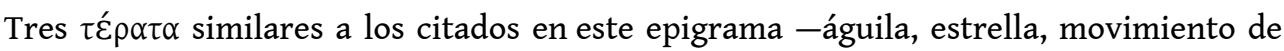
una estatua- son mencionados varios siglos después en la Novela de Alejandro (III, 33), de Ps.-Calístenes, en el pasaje que describe la muerte del rey ${ }^{39}$. Un interesante aspecto este, ya que los tres prodigios que en el epigrama de Posidipo son signos de victoria, en Ps.-Calístenes lo son de muerte. Las diferencias también son obvias. Así pues, en los omina de este ep. 31 A.-B. se podría reconocer una traza del núcleo de la novela griega de Alejandro, que se habría gestado en tiempos de Ptolomeo I ${ }^{40}$. Es posible que Posidipo mencione presagios menos conocidos, tal vez inventados, pero que también reflejaban la práctica adivinatoria efectiva ${ }^{41}$. En cualquier caso, los ponía al servicio de la ideología ptolemaica, como el resto de la leyenda de Alejandro Magno.

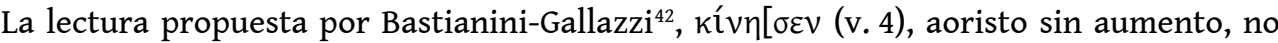
parece necesaria, toda vez que para la estructura del pentámetro nada habría impedido

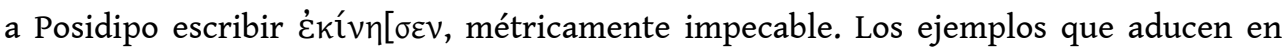
favor de su lectura Bastianini-Gallazzi no son válidos, ya que métricamente no son viables con aumento (ep. 36.7; 39.4; 57.4; 74.5 A.-B.). Por lo tanto, es preferible entender la lectura propuesta por Lapini ${ }^{43}: \kappa i v \eta[\theta \varepsilon ́ v$, como ha quedado visto en mi traducción del dístico (supra).

Por otra parte, hay que señalar que el epigrama tiene una estructura tripartita: en el

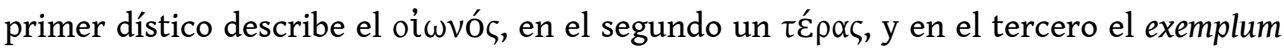
del cumplimiento del augurio. A la unicidad de Alejandro (v.5) se contrapone la condición innumerable de los ejércitos persas (v.6), hecho que realza el carácter portentoso del acontecimiento y del presagio. Por otra parte, la ambivalencia del verbo

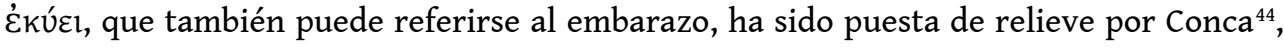
quien ha visto una posible alusión al nacimiento de Alejandro, pues cuenta Plutarco ${ }^{45}$ 
que Olimpíade, la víspera de sus nupcias con Filipo, soñó que un rayo impactaba en su vientre, prendiendo un gran fuego.

11 La primera lectura de Bastianini-Gallazzi (2001), oĩov: "un prodigio tal...", puede ser sustituida por la de Lapini ${ }^{46}$, õ̃ov adverbial, es decir, no se trata de una oposición entre otros reyes y los Argéadas, sino entre los Argéadas en general y uno de ellos en particular: Alejandro ${ }^{47}$. Esto supone quitar la puntuación al final del v. 4 y entender $\delta \varepsilon$ como adversativa que contrapone los oíwvoí del águila y del relámpago, comunes a

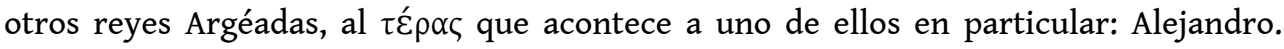
Dicho de otro modo, mientras que a los demás monarcas macedonios se les presentaban los mismos augurios, Alejandro, cuando iba a invadir Persia, tuvo un presagio diferente e irrepetible, que sería uno de los diversos casos en que, según la tradición, el macedonio tuvo relaciones de carácter único con el mundo sobrenatural. En este sentido, Diodoro de Sicilia ${ }^{48}$, Plutarco ${ }^{49}$ y Arriano ${ }^{50}$, que no citan los prodigios contenidos en el ep. 31 A.-B., recuerdan al unísono que la estatua de Orfeo en Libetra, de madera de ciprés, tuvo una exudación, como presagio de buenos augurios para Alejandro y la campaña que iba a emprender ${ }^{51}$, y Calístenes de Olinto ( $\left.F g r H, 124 \mathrm{~F} 14 \mathrm{a}\right)$

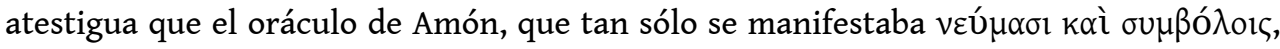
por vez primera rompió su silencio ante la presencia de Alejandro y proclamó la naturaleza divina de éste. Pero, en cualquier caso, como señala Calímaco en el prólogo de sus Aitia (fr. I, 26 Pfeiffer), el de Pela parece obedecer el consejo de Apolo y su carro

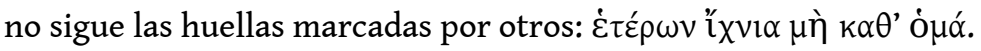

Por otra parte, en el ep. 35 A.-B. podemos leer:

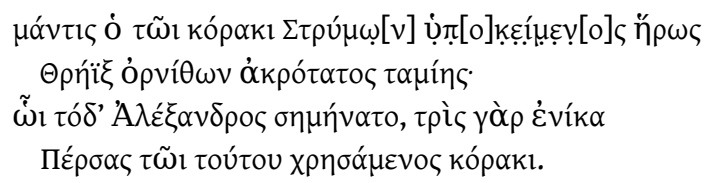

Adivino era el héroe Estrimón el tracio, que yace bajo el cuervo, el mejor dispensador de augurios;

a éste Alejandro le otorgó este signo, pues tres veces venció a los persas tras haber consultado a su cuervo.

Este epigrama es como una inscripción funeraria, casi un epitafio a Estrimón, a quien

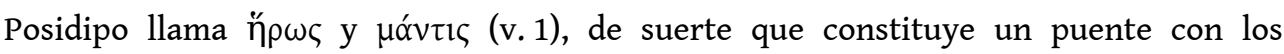
epigramas siguientes, que son funerarios. Está estructurado en dos partes: en el primer dístico señala a quién está dedicado el poema; en el segundo pone en un primer plano a

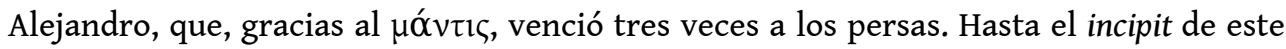

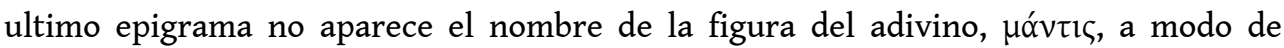
colofón de los epigramas oi $\omega v o \sigma \kappa o \pi ı \alpha ́$, para recordar al lector que la interpretación de los augurios son patrimonio de los adivinos, que son los depositarios de las competencias técnicas pertinentes. En este caso concreto sabemos que se llamaba Estrimón, escasamente documentado como nombre de persona y que era de Tracia, como el río del mismo nombre que en otro tiempo marcó la frontera entre Macedonia y Tracia hasta Filipo II, pero nada más. En el ep. 34 A.-B. aparece Damón, que era el augur preferido de Alejandro, pero no deja de sorprender que un personaje como Estrimón, que, según se deduce del epigrama 35 A.-B., influyó tanto en el macedonio, no haya dejado ni una sola referencia en toda la literatura antigua. No obstante, tampoco hay que conceder demasiada importancia a este hecho, toda vez que sabemos que Alejandro

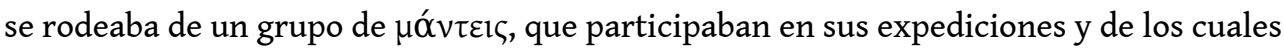


tan sólo tenemos el nombre de algunos de ellos ${ }^{52}$. Este epigrama sintoniza más con las inscripciones funerarias del helenismo tardío y debe de tratarse de un augur ya

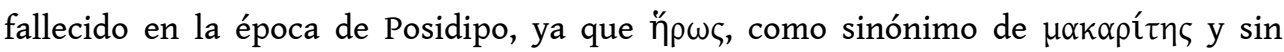
ninguna relación con el heroísmo, aparece con este sentido en los mencionados epígrafes y en el "nuevo" Posidipo designa normalmente a los muertos (ep. 32.5 A.-B.) ${ }^{53}$; tal vez se le tributase algún culto local. Sin embargo, sus predicciones para Alejandro deben ubicarse, como mucho, en torno al 331 a.C. (batalla de Gaugamela), una veintena de años antes del nacimiento de Posidipo ${ }^{54}$. De no tratarse de un mero ejercicio literario, probablemente fuera una inscripción o un epitafio para acompañar a una estatua. En este sentido, Schröder ${ }^{55}$ cree que realmente estamos ante un epitafio, toda

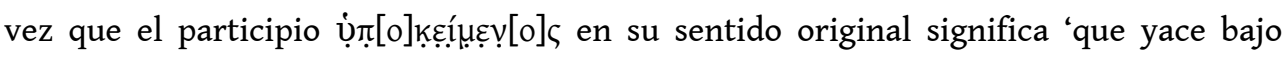
algo', lo que invita a pensar que había una escultura con forma de cuervo -un $\sigma \tilde{\eta} \mu \alpha-$ sobre la tumba del adivino. Esto explicaría mejor la presencia del artículo ò referido a dicho participio y del demostrativo $\tau o ́ \delta(\varepsilon)$ (v. 3). En cualquier caso, este epigrama, al igual que el ep. 31 A.-B., parece vincular a Alejandro y su legado con el pasado Argéada ${ }^{56}$, pues habría sido el macedonio quien, en homenaje a Estrimón, habría erigido esta representación del cuervo $^{57}$. También hay que señalar que existen epigramas funerarios en los que se describen esculturas de animales en las tumbas, incluidas aves $^{58}$. De igual modo, Plutarco, en la Vida de Cicerón (XXVI, 11), cuenta que el orador

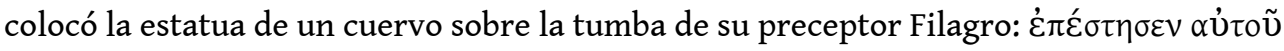

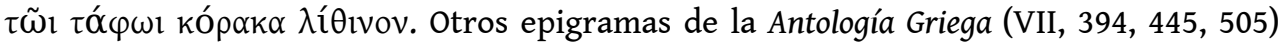
muestran que en algunas tumbas estaban representados los instrumentos de trabajo del

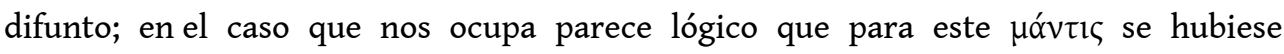
esculpido un cuervo. De manera similar, se pueden ver en otros autores las imágenes ( $\sigma \eta ́ \mu \alpha \tau \alpha)$ grabadas en los escudos de los guerreros argivos reflejando su carácter ${ }^{59}$.

En cuanto a $\tau \tilde{\omega} \iota$ kópakı (v. 1), es sabido que el cuervo es un ave sagrada de Apolo y apta para la adivinación, como se puede leer en Hesíodo ${ }^{60}$, en Eliano ${ }^{61}$, en Bianor ${ }^{62}$ y en Porfirio ${ }^{63}$, así como en la versión del mito a cargo de Ovidio ${ }^{64}$. En la comedia aristofánica los Telmesios (fr. 550 Kassel-Austin) hay una posible alusión a la capacidad augural de los cuervos. También Arato ${ }^{65}$ se refiere a los cuervos como aves cuyo comportamiento es útil para la predicción de lluvia. La mención de kópakı al principio y al final confiere al epigrama una estructura en anillo.

Por otra parte, el encabalgamiento del adjetivo $\Theta p$ ńił muestra la consideración que este

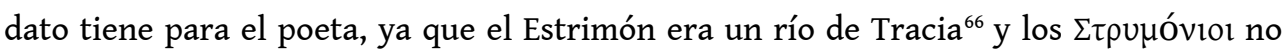
eran sino los tracios ${ }^{67}$. En este sentido, hay otra hipótesis, la propuesta por LavigneRomano ${ }^{68}$, según la cual el nombre de Estrimón haría referencia al dios-río tracio, padre de Reso y que, presuntamente, habría podido practicar la hidromancia, pero que me

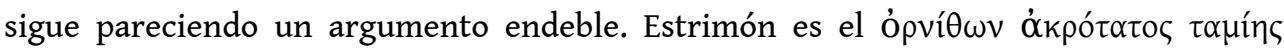
(v. 2), el sumo distribuidor o árbitro de augurios, de manera similar a como Zeus es en la Ilíada (IV, 84) el árbitro de los combates entre los humanos. La alusión al texto homérico viene confirmada en el segundo dístico, donde Alejandro utiliza al cuervo y a la pericia de Estrimón para prever el resultado de su enfrentamiento con los persas. Recordemos a este propósito que la ornitomancia u oionística, como la llama Platón en el Fedro (244C-D), era probablemente la forma más antigua de adivinación en Grecia ${ }^{69} \mathrm{y}$ que toda la vida del macedonio está jalonada de presagios alados, según los testimonios de Diodoro de Sicilia, Plutarco y Arriano. 


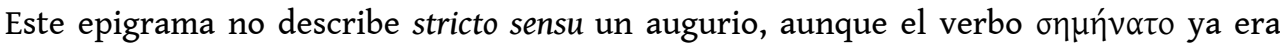
empleado desde Heráclito ${ }^{70}$ para indicar las señales que los dioses facilitaban a los mortales y al serle adjudicado aquí a Alejandro Magno de alguna manera se está sugiriendo por parte del poeta que el macedonio se está atribuyendo un papel reservado a los dioses ${ }^{71}$. En este sentido, Posidipo cuenta que Alejandro 'señaló', esto es, distinguió con un $\sigma \tilde{\eta} \mu \alpha$ a Estrimón, al que había distinguido con el título de ópví $\theta \omega v$

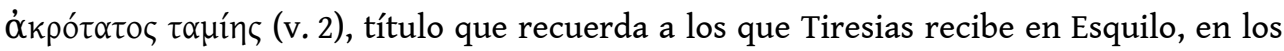

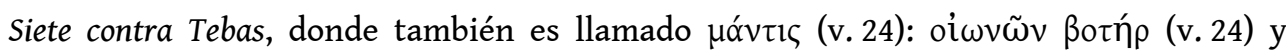

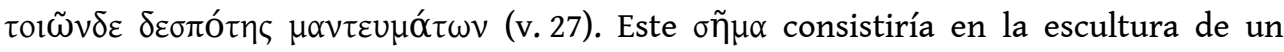
cuervo sobre su tumba, que mantendría vivo el recuerdo de la pericia del adivino y de las victorias de Alejandro. Por otra parte, Posidipo jugaría una vez más con la

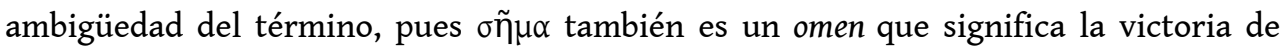
Alejandro, y onuńvato hace, pues, alusión tanto al signo como a la tumba ${ }^{72}$. No obstante, al no ser habitual el uso de la voz media en la significación de este verbo, Lapini ${ }^{73}$ ha

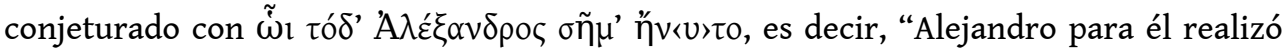
esta tumba", recurriendo al significado que también tiene oñ $\mu \alpha$ como sepulcro, de manera que, en este sentido, este epigrama podría pertenecer tanto a la sección de

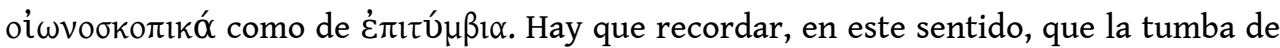
Alejandro estaba en un lugar que probablemente ya se denominaba $\Sigma \tilde{\eta} \mu \alpha$ y que podría tener una conexión con la facultad de onpuíveıv. Fuentes antiguas denominan de esta manera la tumba de Alejandro a partir de la erección de un templo para él y los Ptolomeos por parte de Ptolomeo IV, pero lo más probable es que el primer lugar en el que estuvo depositado el cuerpo de Alejandro, en Alejandría, ya fuese conocido por el nombre de $\Sigma \tilde{\eta} \mu \alpha$ en tiempos de Posidipo y de Ptolomeo II Filadelfo ${ }^{74}$. El adivino Aristandro había profetizado, según cuenta Eliano ${ }^{75}$, que aquel de los Diádocos que diese sepultura a Alejandro en su territorio, se vería gratificado con un poder eterno, predicción que habría incitado a Ptolomeo a transportar su cuerpo a la satrapía de Egipto $^{76}$.

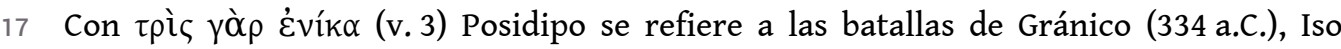

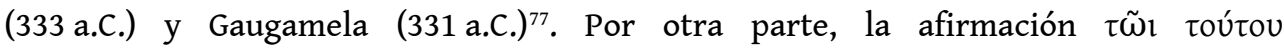

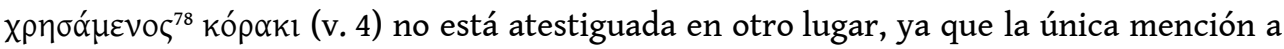
los cuervos que conservamos está relacionada con el viaje de Alejandro al santuario de Amón, en el oasis de Siwa, durante el invierno del 332/331 a.C. ${ }^{79}$ y durante el retorno a Babilonia, en la primavera del 323 a.C., cuando apareció una bandada de cuervos que se picotearon entre sí, cayendo muertos muchos de ellos, presagio del próximo fallecimiento del macedonio ${ }^{80}$. Tanto en el ep. 31 A.-B. como en el ep. 35 A.-B., Alejandro y sus adivinos interpretan correctamente los augurios, de lo que se colige una campaña militar bajo la protección divina, protección que se extiende hasta los Ptolomeos, sus herederos legítimos ${ }^{81}$.

18 Conclusión. El papiro milanés del "nuevo" Posidipo aporta una sección de epigramas con una temática que nos era desconocida y que aporta una tipología completamente nueva, en la que las alusiones históricas y políticas constituyen uno de los rasgos de mayor interés ${ }^{82}$. En ella ocupan un interesante lugar los augurios que se producen en torno a la figura de Alejandro Magno, no sólo por lo novedoso de los augurios en sí, que tienen un contenido inédito en otras fuentes literarias sobre la vida del macedonio, sino también por el hecho de que está presente la vertiente del epigramista como el poeta doctus que maneja información poco conocida, datos no divulgados, olvidados o 
ignorados por la biografía y la historiografía posterior, así como recursos literarios característicos de la poesía helenística como la variatio. No es casualidad que los dos

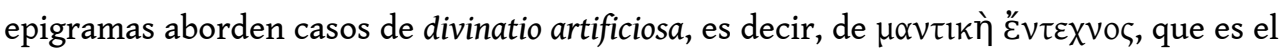
método de adivinación que requiere del concurso de una técnica ( $\tau \varepsilon ́ x v \eta)$, de una pericia conjetural en la interpretación de los signos externos que sólo los adivinos conocían ${ }^{83}$,

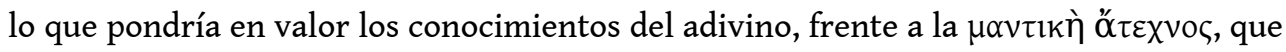
sólo precisa de la inspiración ${ }^{84}$, al tiempo que pone en valor la importancia que tiene la

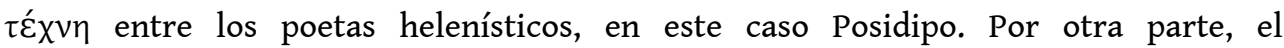
protagonismo que en el ep. 35 adquiere Estrimón, adivino del que hasta el momento no se tenían noticias y que, sin embargo, fue capaz de pronosticar para Alejandro tres victorias sobre los persas, confiere un toque añadido de novedad que no debe pasar desapercibido. En estos dos epigramas también quedan en evidencia algunos de los criterios fundamentales que inspiran a la poesía docta, es decir, la adhesión a la realidad, el gusto por el detalle particular y por la curiositas, así como el deseo de no apartarse de la $\dot{\alpha} \lambda \hat{n} \theta \varepsilon ı \alpha$. El poeta pone de relieve la capacidad técnica de los expertos

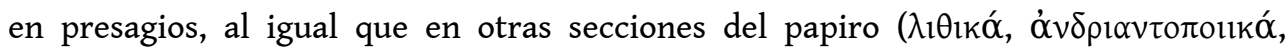

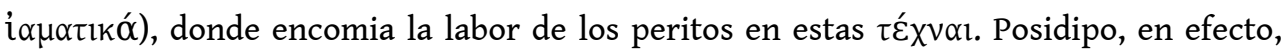
realza prodigios desconocidos, es el poeta savant que sigue "sendas sin trillar",

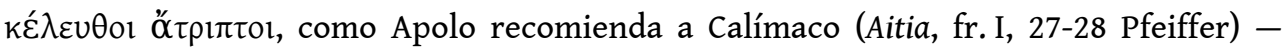

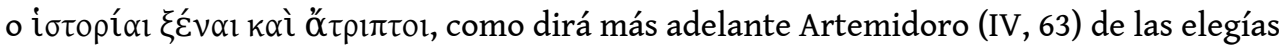
de Partenio de Nicea- y, a la vez, resalta la $\tau \dot{x} \chi v \eta$ empleada en su interpretación. En definitiva, los epigramas estudiados ponen de relieve el carácter excepcional y singular de unos prodigios, de mirabilia en definitiva, ligados a un personaje que todo el mundo antiguo consideró también excepcional.

\section{BIBLIOGRAFÍA}

ANGIÒ Francesca, «Il nuovo Posidippo (2018)», SEP, 16, 2019, pp. 71-104.

AUSTIN Colin \& BASTIANINI Guido, Posidippi Pellaei quae supersunt omnia, Milano, Edizioni Universitarie di Lettere Economia Diritto, 2002.

BASTIANINI Guido \& GALLAZZI Claudio (con la collaborazione di Austin Colin), Posidippo di Pella: Epigrammi (P. Mil. Vogl. VIII 309), Papiri dell'Università degli Studi di Milano, 8, Milano, Edizioni Universitarie di Lettere Economia Diritto, 2001.

BARBANTANI Silvia, «Alexander's Presence (and Absence) in Hellenistic Poetry», en C. Bearzot \& F. Landucci (edd.), Alexander's Legacy (Atti del Convegno dell'Università Cattolica del Sacro Cuore, Milano, settembre 2015), Roma, L'Erma di Bretschneider, 2016, pp. 1-24.

BARBANTANI Silvia, «"His $\sigma \tilde{\eta} \mu \alpha$ Are Both Continens". Alexander the Great in Hellenistic Poetry», Studi Ellenistici, 31, 2017, pp. 51-138.

BAUMBACH Manuel \& TRAMPEDACH Kai, «'Winged Words': Poetry and Divination in Posidippus' Oiônoskopika», en B. Acosta-Hughes, E. Kosmetatou \& M. Baumbach (edd.), Labored in Papyrus 
Leaves: Perspectives on an Epigram Collection Attributed to Posidippus (P. Mil. Vogl. VIII 309), Harvard, Harvard University Press, 2004, pp. 123-160.

BING Peter, «The Politics and Poetics of Geography in the Milan Posidippus, Section One: On Stones (P. Mil. Vogl. VIII 309, col. I-IV 6)», en K. J. Gutzwiller (ed.), The New Posidippus: A Hellenistic Poetry Book, Oxford, Oxford University Press, 2005, pp. 119-140.

BLOCH Raymond, Los prodigios en la Antigüedad clásica, Buenos Aires, Editorial Paidós, 1968 (Paris, Presses universitaires de France, 1963).

BOUCHÉ-LECLERCQ Auguste, Histoire de la divination dans l'Antiquité, Grenoble, Millon, 2003 (Paris, 1879-1882).

CALDERÓN DORDA Esteban, «La "portentosa” vida de Alejandro Magno: visiones, prodigios y presagios en la Vita Alexandri de Plutarco», REA, 115, 2013, pp. 463-476.

CONCA Fabrizio, «Alla ricerca di un poeta», en G. Bastianini \& A. Casanova (edd.), Il papiro di Posidippo un anno dopo, Firenze, Istituto Papirologico «G. Vitelli», 2002, pp. 21-31.

CORTESI Lorenzo, Il mondo dei Tolomei nella grande visione artistico-letteraria di Posidippo di Pella, Torino, Ananke, 2012.

DEFRADAS Jean, «La divination en Grèce», en A. Caquot \& M. Leibovici (edd.), La divination, vol. 1, Paris, Presses universitaires de France, 1968, pp. 157-195.

FERRARI Franco, «Per il testo di Posidippo», M\&D, 54, 2005, pp. 185-212.

FRANCHI Caterina F., «Posidipp. 31 A.-B.», Eikasmós, 16, 2005, pp. 165-167.

FREDRICKSMEYER Ernest A., «Alexander's Religion and Divinity», en J. Roisman (ed.), Brill's Companion to Alexander the Great, Leiden / Boston, Brill, 2003, pp. 253-278.

GIANNINI Alessandro, «Studi sulla paradossografia greca», RIL, 97, 1963, pp. 247-266.

GREENWALDT William S., «A Macedonian Mantis», AncW, 5, 1982, pp. 17-25.

GRONEWALD Michael, «Bemerkungen zum neuen Poseidippos», ZPE, 137, 2001, pp. 1-5.

GUICHARD Luis A., «Posidipo y los prodigios. Una interpretación de P. Mil. Vogl. VIII 309», SEP, 3, 2006, pp. 121-133.

GUTZWILLER Kathryn J., "The Literariness of the Milan Papyrus, or "What Difference a Book?"», en K. J. Gutzwiller (ed.), The New Posidipppus: A Hellenistic Poetry Book, Oxford, Oxford University Press, 2005, pp. 287-319.

KOSMETATOU Elizabeth, «Constructing Legitimacy. The Ptolemaic "Familiengruppe" as Means for Self-Definition in Posidippus' Hippika», en B. Acosta-Hughes, E. Kosmetatou \& M. Baumbach (edd.), Labored in Papyrus Leaves: Perspectives on an Epigram Collection Attributed to Posidippus (P. Mil. Vogl. VIII 309), Harvard, Harvard University Press, 2004a, pp. 225-246.

KOSMETATOU Elizabeth, «Vision and Visibility. Art Historical Theory Paints a Portrait of New Leadership in Posidippus 'Andriantopoiika'», en B. Acosta-Hughes, E. Kosmetatou \& M. Baumbach (edd.), Labored in Papyrus Leaves: Perspectives on an Epigram Collection Attributed to Posidippus (P. Mil. Vogl. VIII 309), Harvard, Harvard University Press, 2004b, pp. 187-211.

LANDUCCI GATTINONI Franca L., «L'indovino Aristandro e l'eredità dei Telmessi», en M. Sordi (ed.), La profezia nel mondo antico, Milano, Pubblicazioni dell'Università Cattolica, 1993, pp. 123-138.

LAPINI Walter, «Il prodigio di Atena (Posidippo, P. Mil. Vogl. VIII 309, col. V 20-25)», ZPE, 141, 2002, pp. 109-110. 
LAVIGNE Donald E. \& ROMANO Allen J., «Reading the Sings. The Arrangement of the New Posidippus Roll (P. Mil. Vogl. VIII 309, IV.7-VI.8)», ZPE, 146, 2004, pp. 13-24.

LLOYD-JONES Hugh, «Posidippus Fr. 31 Austin-Bastianini (P. Mil. Vogl. VIII 309, col. V 20-25)», ZPE, 144, 2003, p. 62.

MÜLLER Sabine, «Poseidippos of Pella and the Memory of Alexander's Campaigns at the Ptolemaic Court», en W. Heckel, S. Müller \& G. Wrightson (edd.), The Many Faces of War in the Ancient World, Cambridge, Cambridge Scholars Publishing, 2015, pp. 135-165.

OGDEN Daniel, Alexander the Great. Myth, Genesis, Sexuality, Exeter, University of Exeter Press, 2011.

PICARD Pierre, «Le portrait de Ptolémée I ou comment construire la monnaie d'un nouveau royaume», CEA, 49, 2012, pp. 19-41.

PRANDI Luisa, «Gli oracoli sulla spedizione asiatica di Alessandro», Chiron, 20, 1990, pp. 345-369.

PRIOUX Évelyne, «Les "Poèmes sur les bronziers” de Posidippe de Pella : traduction et réflexions autour de cinq articles récents», Perspective, 1, 2007a, pp. 48-53.

PRIOUX Évelyne, Regards alexandrins. Histoire et théorie des arts dans l'épigramme hellénistique, Leuven / Paris / Dudley (MA), Peeters, 2007b.

PRIOUX Évelyne, Petits musées en vers. Épigramme et discours sur les collections antiques, Paris, CTHS/ INHA, 2008.

PRIOUX Évelyne, «Les mythes thraces et leurs enjeux politiques dans quelques sources littéraires $\mathrm{du} \mathrm{III}^{\mathrm{e}}$ siècle avant J.-C.», en C. Cusset, N. Le Meur-Weisman \& F. Levin (edd.), Mythe et pouvoir à l'époque hellénistique, Leuven / Paris / Walpole (MA), Peeters, 2012, pp. 109-136.

PRIOUX Évelyne, «Posidippe de Pella, quinze ans plus tard : vers une interprétation d'ensemble du papyrus de Milan», en K. Chryssanthaki-Nagle, K. Descamps-Lequime \& S. Guimier-Sorbets (edd.), La Macédoine du ve siècle avant J.-C. à la conquête romaine. Formation et rayonnement d'une monarchie grecque, Paris, Boccard, 2016, pp. 33-47.

PRIOUX Évelyne, «Parcours poétiques de Posidippe», LALIES, 38, 2018, pp. 303-330.

ROSSI Laura, «Composition and Reception in AP 9.1-583: Aphegheseis, Epideixeis and Progymnasmata», en M. A. Harder, R. F. Regtuit \& G. C. Wakker (edd.), Hellenistic Epigrams, Leuven, Peeters, 2002, pp. 151-174.

SCHRÖDER Stephan, «Überlegungen zu zwei Epigrammen des neuen Mailänder Papyrus», ZPE, 139, 2002, pp. 27-29.

SQUILLACE Giuseppe, «Propaganda macedone e spedizione asiatica: responsi oracolari e vaticini nella spedizione di Alessandro Magno tra verità e manipolazione (nota a Polyaen., Strat., IV, 3, 14)», LEC, 73, 2005, pp. 303-318.

STEINER Deborah T., Images in Mind. Statues in Archaic and Classical Greek Literature and Thought, Princeton, Princeton University Press, 2001.

STEPHENS Susan, «For You, Arsinoe», en B. Acosta-Hughes, E. Kosmetatou \& M. Baumbach (edd.), Labored in Papyrus Leaves: Perspectives on an Epigram Collection Attributed to Posidippus (P. Mil. Vogl. VIII 309), Harvard, Harvard University Press, 2004, pp. 161-176.

TAMMARO Vinicio, «Note posidippee», Eikasmós, 16, 2005, pp. 169-172.

THOMPSON D'Arcy Wentworth, A Glossary of Greek Birds, Hildesheim, Georg Olms AG Verlag, 1966 (Oxford, 1895). 


\section{NOTAS}

1. En Plutarco (Vida de Alejandro, XXXI, 4) aparece el término oi $\omega v o ́ \varsigma$ con el sentido de 'presagio, augurio' (etimológicamente, signo dado por las aves). Sobre la evolución del término, cf. BouchéLeclercq $(2003,108-111)$ y Bloch $(1968,13-14)$.

2. Austin \& Bastianini (2002). Un reciente resumen de la aportación que ha supuesto el descubrimiento y publicación del papiro de Milán puede verse en Prioux (2016).

3. En general, se puede ver Fredricksmeyer (2003) y Calderón Dorda (2013). Es especialmente elocuente un pasaje, a saber, Plutarco, Vida de Alejandro, LXXV, 1-3: ó $\delta$ ' oũv Ad $\lambda \varepsilon^{\prime} \xi \alpha v \delta \rho \circ \varsigma \dot{\omega} \varsigma$

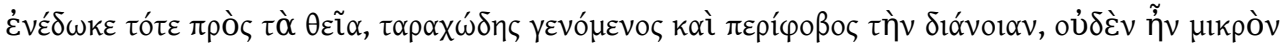

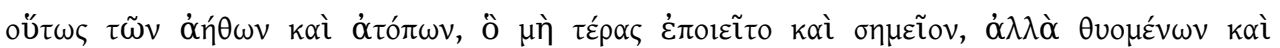

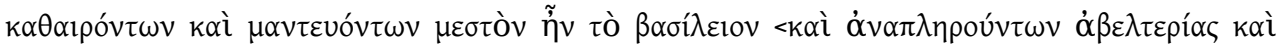

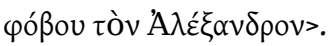

4. Cf. Fredricksmeyer (2003, 256-260) y Calderón Dorda (2013, 475-476).

5. Bing $(2005,119-140)$.

6. Se ha pensado a veces que la estatua de Alejandro aquí descrita era la conocida como Alejandro con lanza, cf. Prioux (2007a, 53, y posteriormente en 2008), pero no hay ninguna certeza de que así sea, cf. Angiò $(2019,92)$. Esta estatua es también distinta de la mencionada en el ep. 70 A.-B., cuyo maltrecho estado no permite extraer conclusiones.

7. Cf. Kosmetatou (2004b, 195-196). Alejandro es objeto de las nuevas tendencias que operan tanto en la poesía (el epigrama), como en las innovaciones de la nuevas tendencias de la escultura (Lisipo) (cf. Barbantani, 2016, 8-9). En Barbantani (2017) se puede ver un repaso de los ecos de Alejandro Magno en la poesía helenística; en concreto, para Posidipo en las pp.90-101. Con carácter general, se puede ver el libro de Cortesi (2012); también son útiles las obras de Prioux (2007b y 2008).

8. Cf. Bouché-Leclercq (2003, 111); Thompson $(1966,7-8)$.

9. Cf. Bouché-Leclercq (2003, 157-159).

10. Cf. Esquilo, Agamenón, 115-117.

11. IV , 15, 26-28.

12. Cf. Plutarco, Vida de Alejandro, XXXIII, 2. Según el pasaje plutarqueo, fue Aristandro quien hizo notar la presencia del águila en dicho acontecimiento, cf. Calderón Dorda $(2013,472)$.

13. Idilios, XVII, 72.

14. Posthoméricas, XII, 56-58.

15. Cf. Stephens (2004, 166, n. 19; 2005, 234 y 237); Kosmetatou (2004a, 242); Picard (2012, 24-25); Barbantani $(2016,6)$.

16. Curcio Rufo, IX, 8, 22; Pausanias, I, 6, 2.

17. Müller $(2015,153)$.

18. Antología Griega, IX, 10, 4.

19. Aristófanes, Paz, 42; Licofrón, Alejandra, 1370; cf. Bouché-Leclercq $(2003,159)$.

20. Cf. Ogden $(2011,84)$.

21. Bastianini \& Gallazzi $(2001,145)$.

22. Lapini $(2002,110)$.

23. Ferrari $(2005,197)$.

24. Un prodigio es siempre una irrupción de lo sagrado en lo profano, Bloch $(1968,10)$. Los prodigios en Posidipo han sido estudiados de manera transversal por Guichard (2006). Una clasificación de los distintos tipos de hechos prodigiosos puede verse en Giannini (1963). Antes del descubrimiento del papiro de Milán se consideraba que los epigramas con tema paradoxográfico eran tardíos y escasos (Rossi, 2002, 164-166), pero los nuevos epigramas de Posidipo parecen demostrar lo contrario (Guichard, 2006, 129). Como señala Aristóteles (Poetica, 


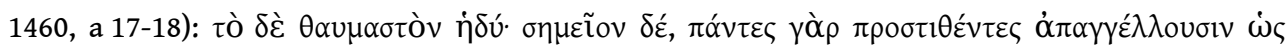

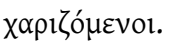

25. Cf. Plutarco, Morales, 397E; Timoleón, XII, 5; Dión Casio, IL, 33, 2; 54.7.3; Virgilio, Eneida, II, 171-175; Tito Livio, XL, 59, 7; Valerio Máximo, I, 6, 12; Suetonio, Calígula, LVII, 1. Sobre el valor augural de las estatuas semovientes, cf. Steiner (2001, $156 \mathrm{ss.})$.

26. Cf. César, Guerra Civil, III, 105, 3; Tácito, Historias, I, 86; Plutarco, Vida de Otón, IV, 8.

27. Bouché-Leclercq (2003, 113-115). Recordemos a este propósito el ep. 26.4 A.-B. de Posidipo, donde utiliza la expresión $\alpha \hat{\gamma} \alpha \theta \tilde{\omega}_{\imath} \sigma \mathrm{U}\langle v\rangle \pi$ róí, completamente novedosa en griego y que sólo volvemos a encontrar en autor bizantino del s. XII, Nicéforo Basilaques (Progymnásmata, LVI, 12).

28. Müller $(2015,154)$.

29. Lapini $(2002,110)$.

30. Schröder (2002, 28-29).

31. Tammaro $(2005,170)$.

32. Bastianini \& Gallazzi $(2001,145)$.

33. Cf. Calímaco, fr. 592 Pfeiffer; Bouché-Leclercq (2003, 547); Barbantani $(2017,93)$.

34. Vida de Alejandro, XIV, 6.

35. Lapini $(2007,222)$.

36. Tammaro $(2005,169-170)$.

37. Gronewald (2001, 2-3).

38. Vida de Alejandro, $\mathrm{XV}, 7$.

39. Cf. Franchi $(2005,166)$.

40. Cf. Barbantani $(2016,15)$.

41. Cf. Baumbach \& Trampedach (2004, 130-131); Müller (2015, 155-156).

42. Bastianini \& Gallazzi $(2001,145)$.

43. Lapini $(2002,109)$.

44. Conca (2002, 30-31).

45. Vida de Alejandro, II, 3.

46. Lapini (2007, 220-222).

47. Barbantani $(2017,93)$. Lloyd-Jones $(2003,62)$ propone oĺwı por oĩov, cf. Ferrari $(2005,196-197)$ y Guichard $(2006,125)$.

48. Biblioteca, XVII, 10, 4 .

49. Vida de Alejandro, XIV, 8.

50. Anábasis, I, 11, 2.

51. Este prodigio infundió temor, pero el adivino Aristandro interpretó el hecho como los sudores y fatigas que las hazañas de Alejandro iban a provocar a los poetas y músicos que las celebrasen (Plutarco, Vida de Alejandro, XIV, 9).

52. Squillace $(2005,313)$.

53. Lapini (2007, 226, n. 78).

54. Cf. Bastianini \& Gallazzi $(2001,149)$.

55. Schröder (2002, 27-28).

56. Cf. Müller $(2015,155)$.

57. Prioux $(2012,119)$.

58. Cf. Antología Griega, VII, 64, 161, 169, 243, 426, 428.

59. Esquilo, Siete contra Tebas, 387, 404, 432, 591, 643; Eurípides, Hécuba, 1273; Ifigenia en Áulide, 239-241.

60. Fr. 60 Merkelbach-West.

61. Sobre la naturaleza de los animales, I, 47-48.

62. Antología Griega, IX, 272.

63. Sobre la abstinencia, III, 5 . 
64. Fastos, II, 243-266.

65. Fenómenos, 963-969.

66. Heródoto, I, 64; Virgilio, Geórgicas, I, 120; Eneida, X, 262-266; Ps.-Plutarco, Sobre los ríos, XI.

67. Heródoto, VII, 75.

68. Lavigne \& Romano $(2004,21)$, tesis recogida de manera más reciente por Prioux (2012, 124-127).

69. Cf. Defradas (1968, 166-170). Cf. Aristófanes, Aves, 708-722.

70. Fr. 22B 93 Diels-Kranz.

71. Gutzwiller $(2005,310)$.

72. Cf. Gutzwiller (2005, 310, n. 70). Véase en Arriano (Anábasis, I, 25, 6-9) un pasaje en el que este mismo verbo es puesto en boca del adivino Aristandro para designar un indicio a partir del comportamiento de un pájaro durante el sitio de Gaza.

73. Lapini $(2007,227)$.

74. Barbantani $(2016,4 ; 2017,54)$.

75. Varia Historia, XII, 64.

76. Cf. Landucci Gattinoni (1993, 129).

77. Sobre la expedición asiática y los oráculos, cf. Prandi (1990).

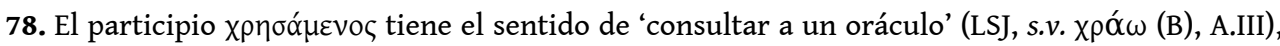

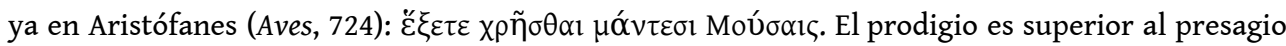
y se impone con más fuerza.

79. Plutarco, Vida de Alejandro, XXVII, 3-4; Arriano, Anábasis, III, 3, 6; Diodoro de Sicilia, Biblioteca, XVII, 49, 5; Estrabón, XVII, 1, 43; Curcio Rufo, IV, 7, 15.

80. Plutarco, Vida de Alejandro, LXXIII, 2.

81. Cf. Müller $(2015,156)$.

82. Cf. Prioux (2016, 39-40) y, en general, Prioux (2018).

83. Cf. Calderón Dorda $(2013,470)$.

84. Sobre los métodos de adivinación, cf. Bouché-Leclercq $(2003,95-96)$. Para Bloch $(1968,22-23)$

el prodigio es superior al presagio y se impone con más fuerza.

\section{RESÚMENES}

A través del estudio de los epigramas 31 y 35 A.-B. de Posidipo, dedicados a Alejandro Magno y a la adivinación, es posible constatar algunos de los principios consagrados de la poesía docta, como son el gusto por lo novedoso y por aquellos detalles que son poco conocidos. En este caso se trata de prodigios de los que no se tenían noticias en las fuentes antiguas y que, además de requerir de un especial conocimiento de la divinatio artificiosa por parte de sus intérpretes, suponen un deseo de realzar el carácter también extraordinario de la figura de Alejandro y de unir su legado a la dinastía de los Ptolomeos.

Par l'étude des épigrammes 31 et 35 A.-B. de Posidippe, dédiées à Alexandre le Grand et à la divination, il est possible de vérifier certains principes consacrés de la poésie docta, comme le goût de la nouveauté et des détails peu connus. Dans ce cas, il s'agit de prodiges dont nous n'avons jamais entendu parler dans les sources antiques et qui, en plus d'exiger une connaissance particulière de la divinatio artificiosa de la part de ses interprètes, impliquent également une 
volonté de vanter le caractère extraordinaire de la vie d'Alexandre et de rattacher son héritage à la dynastie des Ptolémées.

Through the study of epigrams 31 and 35 A.-B. by Posidippus, dedicated to Alexander the Great and to divination, it is possible to verify some of the specific principles of docta poetry, such as the taste for novelty and for little known details. In this case, it is about miracles of which there was no news in the ancient sources, and in addition to requiring special knowledge of the divinatio artificiosa among their interpreters, they also suppose a desire to enhance the extraordinary character of Alexander's life and to unite his legacy with that of the Ptolemaic dynasty.

ÍNDICE

Mots-clés: Posidippe, Alexandre le Grand, divination, épigramme

Palabras claves: Posidipo, Alejandro Magno, adivinación, epigrama

Keywords: Posidippus, Alexander the Great, divination, epigram

\section{AUTOR}

\section{ESTEBAN CALDERÓN DORDA}

Universidad de Murcia

esteban@um.es 